\title{
20. THE ABLATION OF SOLID METEOROIDS
}

\author{
T. R. KAISER and J. JONES* \\ (University of Sheffield, England)
}

\begin{abstract}
Ablation profiles for compact meteoroids have been obtained which take account of the effects of thermal radiation, conduction, meteoroid heat capacity and deceleration, while retaining the simplifying assumption that the onset of massive ablation occurs at a well-defined temperature.

The dependence of the air density, $\rho_{a}$, at the onset of ablation on the initial meteoroid radius, $R_{0}$, is in agreement with the results of Ceplecha and Padevert (1961). For a meteoroid of given composition, velocity and zenith angle there are four critical values for the radius, $R_{1}, R_{2}, R_{3}$ and $R_{4}$. $R_{1}$ is the micrometeorite limit such that if $R<R_{1}$, the ablation temperature is not reached. For $R_{1}<R<R_{2}$ the onset of ablation is delayed due to thermal radiation and occurs at air density $\rho_{a 1}$, which is substantially independent of radius. If $R_{2}<R<R_{3}$ the delay in onset of ablation is due to the finite heat capacity of the meteoroid; it occurs at an air density $\rho_{a 2}$, which is proportional to $R_{0}$, i.e. to $m_{0}{ }^{1 / 3}$, where $m_{0}$ is the initial mass. If $R>R_{3}$ the meteoroid develops a marked thermal gradient and, if it is brittle, will fragment before ablation commences. The smallest fragments will ablate at the air density $\rho_{a 1}$, which is shown to be close to the experimental result of Jacchia et al. (1967) for photographic meteors. If such a particle survives fragmentation, the molten droplet will fragment due to distortion if $R>R_{4}$ (Lebedinec and Portnjagin, 1966); it is shown that $R_{4}$ is not substantially different in magnitude from $R_{3}$. Meteoroids in the size range $R_{2}<R<R_{3}$ produce underdense meteor trains and experimental measurements of echo decay as a function of echo amplitude are in agreement with the expectation that $\rho_{a 2} \propto m_{0}{ }^{n}$ where $n \sim \frac{1}{3}$ (Poole and Kaiser, 1967). This would support the view that the bulk of radio meteors are produced by compact particles and that the fragmentation observed with photographic meteors may be a result of thermal shock.

The ablation profiles have been computed for initial meteoroid velocities between 20 and $70 \mathrm{~km}$ $\mathrm{sec}^{-1}$ and radii between $R_{1}$ and $R_{3}$; the deceleration and residual size (at the end of ablation) have also been calculated. Even quite close to the micrometeorite limit, the residual particle is small compared with the initial size. Hence, meteoroids with $R>R_{1}$ will make negligible contribution to the micrometeorites reaching the Earth's surface, except, perhaps, where a significant proportion of the mass of larger meteoroids fragments into particles with radius less than $R_{1}$. By neglecting deceleration, it is possible to arrive at an analytic expression for the ablation profile; this is found to be in good agreement with the computed profiles except for particles near to the micrometeorite limit.
\end{abstract}

\section{References}

Ceplecha, Z., Padevět, V. (1961) Bull. astr. Inst. Csl., 12, 191.

Jacchia, L.G., Verniani, F., Briggs, R.E. (1967) Smithson. Contr. Astrophys., $10,1$.

Lebedinec, V.N., Portnjagin, Ju. I. (1966) Komety $i$ Meteory, 13, 9.

Poole, L. M. G., Kaiser, T.R. (1967) Planet. Space Sci., 15, 1131.

* Now at University of Western Ontario, Canada.

Kresák and Millman (eds.), Physics and Dynamics of Meteors, 205-206. (c) I.A.U. 


\section{DISCUSSION}

Lebedinec: The first part of Dr. Kaiser's presentation shows how necessary it is to have a regular exchange of information between scientists of different countries. In 1963 formulas and curves of ionization were published by us in the Astronomičeskij Žurnal of the USSR, 40, 719. These are very similar to those presented by Kaiser, and obtained under the same assumptions. In 1963 at the Warsaw COSPAR meeting more accurate formulas and curves were presented, including the effect of the deceleration before and during the process of the evaporation (Space Research, IV).

Sekanina: I should like to ask you whether a possible rotation (or even a more complicated axial motion) of the meteoroid could have any influence on the rate of ablation.

Kaiser: The present calculations, for simplicity, assume spherical bodies. We may perhaps hope that irregular or elongated bodies will, if they rotate, be heated uniformly and behave like a spherical body of a suitable equivalent radius. If there is no rotation the effects of inhomogeneous heating are likely to be greater. 